\title{
PREPROJECTIVE PARTITIONS AND THE DETERMINANT OF THE HOM MATRIX
}

\author{
K. IGUSA ${ }^{1}$ AND G. TODOROV
}

\begin{abstract}
If $\Lambda$ is an artin algebra and ind $\Lambda$ is the set of isomorphism classes of indecomposable finitely generated $\Lambda$-modules, then there is a partition ind $\Lambda=$ $\bigcup_{i \geqslant 0} \underline{P}_{i}$, called the preprojective partition. We give an algorithm for computing this partition, which is given only in terms of numerical properties of the AuslanderReiten quiver of $\Lambda$.

If $\Lambda$ is of finite representation type, then there are two essentially different proofs that the matrix $\operatorname{Hom}=\left(\right.$ length $\left.\operatorname{End}(N) / \operatorname{rEnd}(N)_{1} \operatorname{Hom}_{.}(M, N)\right)$, where $M, N \in$ ind $\Lambda$ has determinant +1 [IT1, W1, Z1]. We show that the paths between the Hom matrix and the identity matrix in $\mathrm{GL}_{n}(\mathbf{R})$ given by these two proofs are homotopic.
\end{abstract}

0. Introduction. If $\Lambda$ is an artin algebra Auslander and Smalø [AS] constructed a partition of the set of isomorphism classes of indecomposable $\Lambda$-modules as ind $\Lambda$ $=\bigcup \underline{\underline{P}}_{i}, i \in \mathbf{N} \cup\{\infty\}$, called the preprojective partition. This partition is characterized by the following properties.

Proposition 0.1 (Auslander And Smal0). (a) $\underline{\underline{P}}_{0}$ consists of the indecomposable projective $\Lambda$-modules.

(b) $\underline{\underline{P}}_{i}$ is finite for each $i<\infty$ and is minimal with respect to the property that each indecomposable module not in $\underline{\underline{P}}_{0}, \ldots, \underline{\underline{P}}_{i-1}$ is a quotient of a direct sum of modules from $\underline{\underline{P}}_{i}$.

(c) $\underline{\underline{P}}_{\infty}$ is the collection of indecomposable modules not in $\bigcup_{i<\infty} \underline{\underline{P}}_{i}$.

A module is called preprojective if it is in $\bigcup_{i<\infty} \underline{\underline{P}}_{i}$.

The first two classes $\underline{\underline{P}}_{0}$ and $\underline{\underline{P}}_{1}$ are easy to determine, but a description of arbitrary $\underline{\underline{P}}_{i}$ given $\underline{\underline{P}}_{0}, \ldots, \underline{\underline{P}}_{i-1}$ involves morphisms between modules. For hereditary artin algebras there is a simple algorithm for determining $\underline{\underline{P}}_{i}$ if $\underline{\underline{P}}_{i-1}$ is known [T]. Also for selfinjective algebras of type $A_{n}$ there is an algorithm for determining the preprojective partition [B]. More details about preprojective partitions for hereditary and algebras stably equivalent to hereditary algebras can be found in [ $\mathbf{Z 2}$ and $\mathbf{W Z}]$. In the first part of this paper we give an algorithm for computing the preprojective

Received by the editors August 18, 1983 and, in revised form, July 5, 1984.

1980 Mathematics Subject Classification. Primary 16A64, 16A46; Secondary 16A60.

Key words and phrases. Indecomposable modules, preprojective partitions, almost split sequences, rings of global dimension 2, Auslander-Reiten quiver.

${ }^{1}$ This author is a Sloan Fellow and is supported by NSF Grant MCS 8202246. 
partition for arbitrary artin algebras using only information given by the Auslander-Reiten quiver.

Original motivation for this paper came from trying to understand the relation between different proofs of the fact that the determinant of the matrix Hom = (length $\operatorname{End}(N) / \operatorname{rEnd}(N) \operatorname{Hom}_{\Lambda}(M, N)$ ) for an algebra of finite representation type is +1 [DF, IT1, W1, Z1], where $\operatorname{rEnd}(N)$ denotes the radical of $\operatorname{End}(N)$ and $M$ and $N$ run through a complete set of the representatives of the isomorphism classes of indecomposable $\Lambda$-modules. In [IT1 and W1] it was shown that there is a path from the inverse of the Hom matrix to the identity matrix. In this paper we show that this path is homotopic to the inverse of the path which is given by factorization of the Hom matrix into elementary matrices as in [Z1]. The proof in $[\mathbf{Z 1}]$ was done by considering algebras of global dimension $\leqslant 2$ and their Cartan matrix, i.e., the matrix (length $\operatorname{End}(Q) / \operatorname{rEnd}(Q) \operatorname{Hom}(P, Q))$, where $P, Q$ run through the isomorphism classes of indecomposable projective modules. For the induction step Zacharia proves the existence of a simple module with projective dimension $\leqslant 1$ and eliminates the projective cover of that module. If $\Lambda$ is of finite representation type with $M_{1}, \ldots, M_{n}$ being representatives of the isomorphism classes of indecomposable $\Lambda$-modules, then the corresponding Auslander algebra $\Gamma=\operatorname{End}\left(\amalg_{i=1}^{n} M_{i}\right)$ has global dimension $\leqslant 2$ and indecomposable projective $\Gamma$-modules correspond to indecomposable $\Lambda$-modules. We show here that the method of eliminating projectives for the Auslander algebra $\Gamma$ can be done in the order of the preprojective partitions of ind $\Lambda$.

1. The algorithm to compute the preprojective partition. Let $\Lambda$ be an artin algebra (not necessarily of finite representation type) and let $U_{j \geqslant 0} \underline{\underline{P}}_{j}$ be the preprojective partition of ind $\Lambda=$ the set of isomorphism classes of indecomposable $\Lambda$-modules. Let $\underline{\underline{C}}_{i}$ be the full subcategory of $\bmod \Lambda$ whose objects lie in $\bigcup_{j \leqslant i} \underline{\underline{P}}_{j}$. We denote by $\bmod \underline{\underline{C}}_{i}^{\mathrm{op}}$ the category of finitely generated contravariant functors from $\underline{\underline{C}}_{i}$ to the category of abelian groups. We will show that $\bmod \underline{\underline{C}}_{i}^{\text {op }}$ has global dimension $\leqslant 2$. Furthermore, if $X \in \underline{\underline{P}}_{i}$ then the simple contravariant functor from $\underline{C}_{i} \subset \bmod \Lambda$ to the category of abelian groups given by $(, X) / r(, X)$ has projective dimension $\leqslant 1$ in $\bmod \underline{\underline{C}}_{i}^{\text {op }}$, where $(, X)=\operatorname{Hom}_{\Lambda}(, X)$. We denote by add $\underline{\underline{C}}_{i}$ the additive subcategory of $\bmod \Lambda$ generated by $\underline{C}_{i}$.

Lemma 1.1. (i) Let $f: Y \rightarrow X$ be a $\Lambda$-homomorphism. If $Y$ is an object in add $\underline{\underline{C}}_{i}$, then im $f$ is an object in add $\underline{\underline{C}}_{i}$.

(ii) If $X$ is a $\Lambda$-module then there exists a unique maximal submodule of $X$ which lies in add $\underline{\underline{C}}_{i}$. We denote it by $\tau_{i} X$. (This is also called the trace of add $\underline{\underline{C}}_{i}$ in $X$.)

(iii) $\left(, \tau_{i} X\right)=(, X)$ as functors on add $\underline{C}_{i}$.

Proof. (i) follows from 0.1(b), and (ii) follows from (i). (iii) follows trivially.

THEOREM 1.2. $\mathrm{gl} \operatorname{dim}\left(\bmod \underline{\underline{C}}_{i}^{\mathrm{op}}\right) \leqslant 2$. 
Proof. If $\underline{\underline{C}}$ is any additive category, then $\operatorname{gl} \operatorname{dim}\left(\bmod \underline{\underline{C}}^{\mathrm{op}}\right) \leqslant 2$ if and only if $\underline{\underline{C}}$ has kernels, since $X \rightarrow(, X)$ is a left exact functor. Let $f: X \rightarrow Y$ be a morphism in add $\underline{\underline{C}}_{i}$. Then $\tau_{i}(\operatorname{ker} f)$ is the kernel of $f$ in add $\underline{\underline{C}}_{i}$. Since $\bmod \underline{\underline{C}}_{i}^{\mathrm{op}}=\bmod$ add $\underline{\underline{C}}_{i}^{\mathrm{op}}$, it follows that $\mathrm{gl} \operatorname{dim}\left(\bmod \underline{\underline{C}}_{i}^{\text {op }}\right) \leqslant 2$.

We now give conditions for a module to be in $\underline{\underline{P}}_{i}$.

Lemma 1.3. Let $B \in \mathrm{Ob} \underline{\underline{C}}_{i}$, let $\alpha: E(B) \rightarrow B$ denote the right minimal almost split map for $B$ with $\operatorname{ker} \alpha=\Delta B$, and let $S=(, B) / r(, B)$. Then the following sequence of functors restricted to add $\underline{\underline{C}}_{i}$ is exact:

$$
0 \rightarrow\left(, \tau_{i} \Delta B\right) \rightarrow\left(, \tau_{i} E(B)\right) \rightarrow(, B) \rightarrow S \rightarrow 0 .
$$

Proof. This follows from the exactness of $0 \rightarrow(, \Delta B) \rightarrow(, E(B)) \rightarrow(, B) \rightarrow S$ $\rightarrow 0$ [AR] and Lemma 1.1(iii).

We will need the following characterization of modules in $\underline{P}_{i}$ : An indecomposable $\Lambda$-module $X$ is in $\underline{\underline{P}}_{i}$ if and only if any epimorphism $Y \rightarrow X$ with $Y \in$ add $\underline{\underline{C}}_{i}$ splits, i.e., $X$ is a splitting projective in add $\underline{C}_{i}$.

THEOREM 1.4. Let $\Lambda$ be an artin algebra, $\cup_{j \geqslant 0} \underline{\underline{P}}_{j}$ the preprojective partition and $\underline{C}_{i}=\bigcup_{j \geqslant i} \underline{P}_{j}$. Let $B \in \mathrm{Ob} \underline{\underline{C}}_{i}$. Then $B \in \underline{\underline{P}}_{i}$ if and only if $r(, B)=(, A)$ in $\bmod \underline{\underline{C}}_{i}^{\mathrm{op}}$ for some $\Lambda$-module $A$ and the induced map $A \rightarrow B$ is a $\Lambda$-monomorphism.

Proof. Suppose $r(, B)=(, A)$ in $\bmod \underline{\underline{C}}_{i}^{\text {op }}$ and $A \rightarrow B$ is a monomorphism:

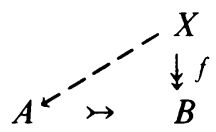

If $X$ is in add $\underline{\underline{C}}_{i}$ and $f: X \rightarrow B$ is a nonsplit epimorphism, then $(, X) \rightarrow(, B) \rightarrow$ $(, B) / r(, B)$ is zero. Consequently $f$ lifts to $A$. But then im $f \subset A \varsubsetneqq B$. Contradiction. Hence $B$ is a splitting projective in add $\underline{C}_{i}$. Therefore $B \in \underline{P}_{i}$.

Now suppose that $B \in \underline{\underline{P}}_{i}$. Then $B$ is a splitting projective in $\underline{C}_{i}$. Consider the following commutative diagram with exact rows:

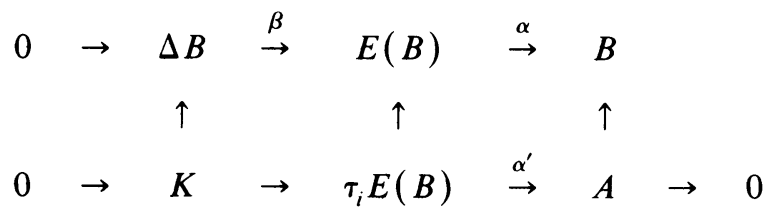

Let $A=\alpha \tau_{i} E(B)$ and $K=\beta^{-1} \tau_{i} E(B)=\operatorname{ker} \alpha^{\prime}$ in the above diagram. Then $A \neq B$, since otherwise $\tau_{i} E(B) \rightarrow B$ is a split epimorphism making $\alpha$ also a split epimorphism. So $A \rightarrow B$ lifts to $E(B)$ and consequently to $\tau_{i} E(B)$. Thus $\alpha^{\prime}$ is a split epimorphism and $K \cong \tau_{i} E(B) / A \in \mathrm{Ob}$ add $\underline{\underline{C}}_{i}$. But $\tau_{i} \Delta B$ goes to zero in $A$ so 
$\tau_{i} \Delta B \subset K$. Thus $K=\tau_{i} \Delta B$. So $\tau_{i} \Delta B \rightarrow \tau_{i} E(B)$ splits and the cokernel $A$ is a submodule of $B$. Then by $1.3,(, A)=r(, B)$.

Corollary 1.5. If $B \in \underline{\underline{P}}_{i}$ let $r_{i} B$ denote the image of $\tau_{i} E(B)$ in $B$, i.e. $\left(, r_{i} B\right)=$ $r(, B)$. Then $\tau_{i} E(B) \cong r_{i} B \amalg \tau_{i} \Delta B$.

Corollary 1.6. Let $B \in \mathrm{Ob} \underline{\underline{C}}_{i}$. Then $B$ lies in $\underline{\underline{P}}_{i}$ if and only if

$$
l(B)+l\left(\tau_{i} \Delta B\right)-l\left(\tau_{i} E(B)\right)>0,
$$

where l denotes the length of a module.

Proof. Suppose $B \in \underline{\underline{P}}_{i}$. Let $A=r_{i} B$. Then $l(B)>l(A)=l\left(\tau_{i} E(B)\right)-l\left(\tau_{i} \Delta B\right)$. Now suppose that $B \notin \underline{\underline{P}}_{i}$. Then there is an $X \in \mathrm{Ob}$ add $\underline{\underline{P}}_{i}$ which maps onto $B$. This map lifts to $E(B)$ and therefore to $\tau_{i} E(B)$, and thus $\tau_{i} E(B)$ maps onto $B$. But $\tau_{i} \Delta B$ is always contained in the kernel of $\tau_{i} E(B) \rightarrow B$. So $l(B)+l\left(\tau_{i} \Delta B\right)-l\left(\tau_{i} E(B)\right) \leqslant 0$.

We now give an algorithm for computing the preprojective partition using 1.6.

Let $K_{0}=K_{0}(\bmod \Lambda, \amalg)$ be the Grothendieck group of the set of isomorphism classes of $\Lambda$-modules (we only consider finitely generated modules) considered as a monoid under direct sum. Then $K_{0}$ is the free abelian group generated by ind $\Lambda$. For an indecomposable $\Lambda$-module $X$ and $i \geqslant 0$ let $g_{i} X=X+\tau_{i} \Delta X-\tau_{i} E(X) \in K_{0}$. Let $M_{i}$ be the integer matrix with rows and columns corresponding to the elements of ind $\Lambda$ so that $g_{i} X=\sum_{Y} M_{i}(Y, X) Y$ where $Y$ runs over ind $\Lambda$. Then the columns of $M_{0}$ are given by right minimal almost split maps for projectives and almost split sequences (Auslander-Reiten sequences) for nonprojectives. These are known to form a dual basis [A, IT1] to the standard basis ind $\Lambda$ with respect to the bilinear form given by $\operatorname{dim}_{\operatorname{End}(N) / \operatorname{rEnd}(N)} \operatorname{Hom}_{\Lambda}(M, N)$. Hence $M_{0}$ is the inverse of the Hom matrix.

LEMMA 1.7. For each $i \geqslant 0$ the matrix $M_{i}$ satisfies the following:

(a) $M_{i}(Y, X)=0$ if $Y \neq X$ and $Y \in \underline{\underline{P}}_{0} \cup \cdots \cup \underline{\underline{P}}_{i-1}$.

(b) $M_{i}(X, X)=1$ if $X \in \underline{\underline{P}}_{0} \cup \cdots \cup \underline{\underline{P}}_{i-1}$.

(c) If $X \in \underline{\underline{P}}_{i}, Y \neq X$ and $M_{i}(Y, X) \neq 0$, then $l(Y)<l(X)$.

Proof. (a) and (b) follow from the equation $g_{i} X=X+\tau_{i} \Delta X-\tau_{i} E(X)=$ $\sum_{Y} M_{i}(Y, X) Y$ and the fact that the indecomposable summands of $\tau_{i} \Delta X$ and $\tau_{i} E(X)$ are elements of $\underline{\underline{C}}_{i}=\underline{\underline{P}}_{i} \cup \underline{\underline{P}}_{i+1} \cup \cdots$.

(c) If $X \in \underline{\underline{P}}_{i}$, then by $1.5, r_{i} X=\tau_{i} E(X)-\tau_{i} \Delta X$, so $g_{i} X=X-r_{i} X$. If $Y \neq X$ and $M_{i}(Y, X) \neq 0$ then $Y \subset r_{i} X \varsubsetneqq X$ so $l(Y)<l(X)$.

Lemma 1.8. If $g_{i} X$ is given, then $g_{i+1} X$ can be obtained by replacing each $Y \in \underline{\underline{P}}_{i}$ which occurs in $g_{i} X-X$ by $r_{i} Y$ and repeating the process for any new summands obtained in this way which lie in $\underline{\underline{P}}_{i}$.

Proof.

$$
\begin{gathered}
g_{i} X=X-\tau_{i} E(X)+\tau_{i} \Delta X, \\
g_{i+1} X=X-\tau_{i+1} E(X)+\tau_{i+1} \Delta X .
\end{gathered}
$$


Since $r_{i} Y \in \mathrm{Ob}$ add $\underline{\underline{C}}_{i}$ and $l\left(r_{i} Y\right)<l(Y)$ the process described must stop with all modules but $X$ being in $\underline{\underline{P}}_{i+1} \cup \underline{\underline{P}}_{i+2} \cup \cdots$. This process gives $g_{i+1} X$ since $\tau_{i+1} r_{i} Y$ $=\tau_{i+1} Y$ for all $Y \in \underline{\underline{P}}_{i}$.

LEMMA 1.9. The matrix $M_{i+1}$ can be obtained from the matrix $M_{i}$ by eliminating all nondiagonal entries in the rows which correspond to modules in $\underline{\underline{P}}_{i}$. Thus $M_{i+1}=M_{i} E$ for some invertible matrix $E$.

Proof. The process described in 1.8 corresponds to performing column operations $e_{Y X}\left(-M_{i}(Y, X)\right)$ on $M_{i}$, where $e_{m n}(c)$ is the elementary matrix with 1 's on the diagonal, $c$ in the $m n$-entry, and 0 's elsewhere.

LEMMA 1.10. Let $V$ be the row matrix giving the lengths of all indecomposable $\Lambda$-modules. Then the $X$ entry of $V M_{i}$ is the length of $g_{i} X$. Furthermore, if $M_{i+1}=M_{i} E$, then $V M_{i+1}=\left(V M_{i}\right) E$.

Defrinition 1.11. Let $N_{i}$ denote the matrix $M_{i}$ with an additional row $V M_{i}$. Thus $N_{i}=\left(\begin{array}{l}l \\ v\end{array}\right) M_{i}$.

Proposition 1.12. Let $N_{i}$ be as above. Then a module $X$ is in $\underline{\underline{P}}_{i}$ if and only if the additional row $V M_{i}$ in the matrix $N_{i}$ has a positive integer in the $X$ position and $X$ does not belong to $\underline{\underline{P}}_{0} \cup \cdots \cup \underline{\underline{P}}_{i-1}$.

Proof. If $X$ is in $\underline{\underline{P}}_{i}$, then, by $1.6, g_{i} X>0$. Conversely, if $g_{i} X>0$, then, by the same corollary, $X \in \underline{\underline{P}}_{0} \cup \cdots \cup \underline{\underline{P}}_{i}$.

This proposition gives us an algorithm for computing the preprojective partition.

Algorithm 1.13. (0) Let $M_{0}$ be the matrix with columns given by right minimal almost split maps. Let $N_{0}$ be the matrix $M_{0}$ with an additional row which has 1's in the projective columns and 0 's elsewhere.

(1) Given $N_{i}$ and $\underline{\underline{P}}_{0}, \ldots, \underline{\underline{P}}_{i-1}$ we get that $\underline{\underline{P}}_{i}$ consists of those $X \notin \underline{\underline{P}}_{0} \cup \cdots \cup \underline{\underline{P}}_{i-1}$ so that the bottom row of $N_{i}$ has a positive number in the $X$ position.

(2) $N_{i+1}$ is obtained from $N_{i}$ by performing column operations to eliminate all nondiagonal entries in the rows corresponding to the elements of $\underline{\underline{P}}_{i}$.

COROLlaRY 1.14. If $\Lambda$ is of finite representation type, the matrix $N_{i}$ becomes $\left(\begin{array}{l}I \\ V\end{array}\right)$, where $V$ is the row matrix whose entries are the lengths of the indecomposable $\Lambda$-modules.

To illustrate the algorithm we give a simple example. Let $\Lambda$ be the selfinjective algebra $\Lambda=k[x] /\left(x^{3}\right)$ with Auslander-Reiten quiver given by:

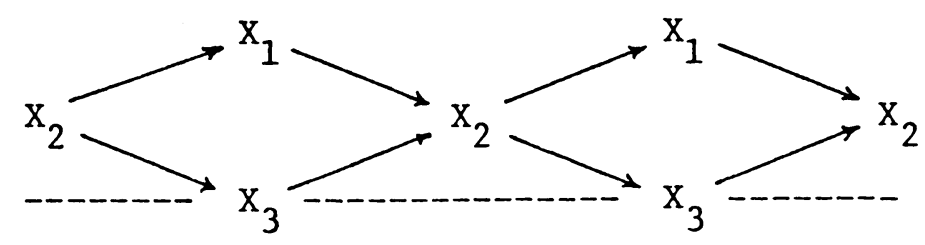




$$
\begin{aligned}
& N_{0}=\left(\begin{array}{ccc}
1 & -1 & 0 \\
-1 & 2 & -1 \\
0 & -1 & 2 \\
1 & 0 & 0
\end{array}\right), \quad \underline{\underline{P}}_{0}=\left\{X_{1}\right\} ; \\
& N_{1}=N_{0} e_{12}(1)=\left(\begin{array}{ccc}
1 & 0 & 0 \\
-1 & 1 & -1 \\
0 & -1 & 2 \\
1 & 1 & 0
\end{array}\right), \quad \underline{\underline{P}}_{1}=\left\{X_{2}\right\} ; \\
& N_{2}=N_{1} e_{23}(1) e_{21}(1)=\left(\begin{array}{ccc}
1 & 0 & 0 \\
0 & 1 & 0 \\
-1 & -1 & 1 \\
2 & 1 & 1
\end{array}\right), \quad \underline{\underline{P}}_{2}=\left\{X_{3}\right\} ; \\
& N_{3}=N_{2} e_{31}(1) e_{32}(1)=\left(\begin{array}{ccc}
1 & 0 & 0 \\
0 & 1 & 0 \\
0 & 0 & 1 \\
3 & 2 & 1
\end{array}\right) \leftarrow \text { lengths of modules. }
\end{aligned}
$$

2. A homotopy between two proofs that the det Hom $=+1$. Throughout this section we shall assume that $\Lambda$ is an artin algebra of finite representation type. Thus $M_{i}=I$ for sufficiently large $i$ and Algorithm 1.13 gives elementary matrices $E_{1}, \ldots, E_{k}$ such that $M_{0} E_{1} \cdots E_{k}=I$. So $M_{0}=E_{k}^{-1} \cdots E_{1}^{-1}$. Since $M_{0}^{-1}$ is the Hom matrix this gives a path

$$
h(t)=\left(t E_{k}^{-1}+(1-t) I\right) \cdots\left(t E_{1}^{-1}+(1-t) I\right)
$$

from $I$ to the matrix $M_{0}=\operatorname{Hom}^{-1}$ in $\mathrm{GL}_{n}(\mathbf{R})$, where $n=\mid$ ind $\Lambda \mid$. The path $h(t)$ is uniquely determined up to homotopy since the elementary matrices $E_{1}, \ldots, E_{k}$ are uniquely determined up to Steinberg relations [M] by 1.7(c). (This is not important since $h(t)$ will be shown to be homotopic to a fixed path.)

There is another description of the inverse of the Hom matrix using the fact that its columns are given by right minimal almost split maps and almost split sequences (Auslander-Reiten sequences). Let $C$ be the $n \times n$ matrix with entries $C_{A B}=\delta_{A \Delta B}$ and let $T$ be given by $T_{A B}=\operatorname{dim}_{\mathrm{End}(A)} \operatorname{Hom}\left((, A), r(, B) / r^{2}(, B)\right)$. Thus $T$ is the matrix of irreducible maps. Then the inverse of the Hom matrix can be written as $\mathrm{Hom}^{-1}=I-T+C$, and in [IT1] it was shown that $I-T t+C t^{2}$ is a path from $I$ to $\mathrm{Hom}^{-1}$ in $\mathrm{GL}_{n}(\mathbf{R})$. In this section we shall show that the paths $I-T t+C t^{2}$ and $h(t)$ are homotopic.

Definition 2.1. Let $X, Y \in$ ind $\Lambda$. Then a $\Lambda$-homomorphism $f: X \rightarrow Y$ is said to have depth $\geqslant i$ if $f$ can be written as a sum of compositions of $i$ or more irreducible maps. The group of all $\Lambda$-homomorphisms $X \rightarrow Y$ of depth $\geqslant i$ is denoted by $r^{i}(X, Y)$.

We shall now restrict ourselves to the following special case. Assume that for all $X, Y \in$ ind $\Lambda$ there exists at most one integer $i$ so that $r^{i}(X, Y) / r^{i+1}(X, Y) \neq 0$. In other words all nonzero maps $X \rightarrow Y$ have the same depth.

Let $K_{0}[t]=K_{0}(\bmod \Lambda, \sqcup)[t]$. For each $X \in$ ind $\Lambda$ and $i \geqslant 0$ let $\hat{\tau}_{i} X \in K_{0}[t]$ be given by $\hat{\tau} X=\sum_{Y} m_{Y} Y t^{k}$ where $\tau_{i} X=\bigsqcup_{Y} m_{Y} Y$ and $k$ is the depth of the composition $Y \subset \tau_{i} X \subset X$. Since $K_{0}[t]$ is the free $Z[t]$-module generated by ind $\Lambda$, we may 
regard $\hat{\tau}_{i}$ as a $\mathbf{Z}[t]$-homomorphism $K_{0}[t] \rightarrow K_{0}[t]$. For each $X \in \underline{\underline{P}}_{i}$ let $\hat{r}_{i} X \in K_{0}[t]$ be given by $\hat{r}_{i} X=\Sigma_{Y} n_{Y} Y t^{k}$, where $r_{i} X=\bigsqcup_{Y} n_{Y} Y$ and $k$ is the depth of $Y \subset r_{i} X \subset X$.

Lemma 2.2. Let $X \in \underline{\underline{P}}_{i}$. Then:

(a) $\hat{r}_{i} X=\hat{\tau}_{i}(E(X)) t-\hat{\tau}_{i}(\Delta X) t^{2}$.

(b) $\hat{\tau}_{i+1} \hat{r}_{i} X=\hat{\tau}_{i+1} X$.

Proof. Suppose that $Y$ is a component of $\tau_{i} E(X) \cong \tau_{i} \Delta X \amalg r_{i} X$ and let $m, a, b$ be the multiplicities of $Y$ in $\tau_{i} E(X), \tau_{i} \Delta X, r_{i} X$, respectively. Then $m=a+b$ and the coefficients of $Y$ in $\hat{\tau}_{i} E(X), \hat{\tau}_{i} \Delta X, \hat{r}_{i} X$ are $m t^{k}, a t^{k-1}, b t^{k+1}$, respectively, for some $k$ by the Radical layers theorem [IT1].

Statement (b) follows from the equation $\tau_{i+1} r_{i} X=\tau_{i+1} X$ and the fact that the depth of $Y \subset Z \subset X$ is the sum of the depths of $Y \subset Z$ and $Z \subset X$ for each component $Z$ of $r_{i} X$ and $Y$ of $\tau_{i+1} Z$. (This uses again the Radical layers theorem.)

For each $X \in$ ind $\Lambda$ and $i \geqslant 0$ let $\hat{g}_{i} X=X-\hat{\tau}_{i}(E(X)) t+\hat{\tau}_{i}(\Delta X) t^{2} \in K_{0}[t]$. Let $\hat{M}_{i} \in \mathrm{GL}_{n}(\mathbf{Z}[t])$ be given by $\hat{g}_{i} X=\sum_{Y} \hat{M}_{i}(Y, X) Y$. Since $\hat{\tau}_{0} X=X$ for all $X$ we have $\hat{M}_{0}=I-T t+C t^{2}$.

Proposition 2.3. Suppose that $\Lambda$ is an artin algebra of finite representation type which satisfies the condition that for all $X, Y \in$ ind $\Lambda$ there exists at most one integer $i$ so that $r^{i}(X, Y) / r^{i+1}(X, Y) \neq 0$. Then $\hat{M}_{0}(t)$ is homotopic to any path $h(t)$ given by Algorithm 1.13 .

Proof. If $X \in \underline{\underline{P}}_{i}$, then 2.2(a) implies $\hat{g}_{i} X=X-\hat{r}_{i} X$. This gives us the analogue of 1.7. Lemma 2.2(b) gives us the analogue of 1.8. Thus $\hat{M}_{0}$ can be written as a product of elementary matrices $e_{i j}(u)$ where $u \in \mathbf{Z}[t]$. If $t=0$, then $\hat{M}_{i}(0)=I$ for all $i$, so $u(0)=0$ in each $e_{i j}(u)$. If $t=1$, then the column operations $e_{i j}(u(1))$ are the ones that transform $\hat{M}_{0}(1)=M_{0}$ to $I$ as in Algorithm 1.13. Thus we have a continuous function $H:[0,1]^{2} \rightarrow \mathrm{GL}_{n}(\mathbf{R})$ given by $H(s, t)=\prod e_{i j}(s u(t))$ which satisfies $H(s, t)=I$ if $s=1$ or $t=0, H(0, t)=\hat{M}_{0}(t)$ and $H(s, 1)=h(1-s)$. Thus $H$ gives a homotopy from $\hat{M}_{0}(t)$ to $h(t)$. Since the nondiagonal entries of $\hat{M}_{i}$ can be eliminated in any order in $1.13(\mathrm{c})$, we can obtain any $h(t)$ :

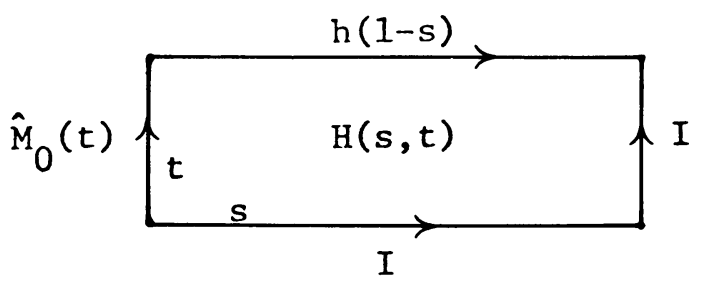

We now consider the general case. Let $\Gamma$ be the modulated Auslander-Reiten quiver of $\Lambda$. In [IT2] it was shown that $\Gamma$ admits a finite covering $p: \tilde{\Gamma} \rightarrow \Gamma$ so that for all $x, y \in \tilde{\Gamma}_{0}$ all nonzero maps $x \rightarrow y$ in the mesh category of $\tilde{\Gamma}$ have the same depth. Then $\tilde{\Gamma}$ is a standard covering and so there is a unique standard algebra $\tilde{\Lambda}$ such that ind $\tilde{\Lambda} \simeq \operatorname{mesh}(\tilde{\Gamma})$. Let $\tilde{\Lambda}$ be the artin algebra associated to $\tilde{\Gamma}$. Then $\tilde{\Lambda}$ 
satisfies the special condition used above, so 2.2 and 2.3 hold for $\tilde{\Lambda}$. Since $\tilde{\Gamma}$ is by construction a normal covering of $\Gamma$ (i.e. corresponds to a normal subgroup of $\pi_{1} \Gamma$ ), the subgroup $G$ of the automorphism group of the underlying valued quiver of $\tilde{\Gamma}$, which preserves fibers, acts transitively on each fiber. Thus each $\tilde{x} \in p^{-1}(x)$ lies in the same preprojective partition $\underline{\underline{P}}_{i}$ of ind $\tilde{\Lambda}$, since the preprojective partition is an invariant of the underlying valued quiver by Algorithm 1.13.

Let $K_{0}[t]=K_{0}($ ind $\Lambda, \amalg)[t]$, let $\tilde{K}_{0}[t]=K_{0}($ ind $\tilde{\Lambda}, \amalg)[t]$, and let $p_{*}: \tilde{K}_{0}[t] \rightarrow$ $K_{0}[t]$ be the $\mathbf{Z}[t]$-homomorphism induced by $p$. For each $X \in$ ind $\Lambda=\Gamma_{0}$ let $\bar{\tau}_{i} X$, $\bar{r}_{i} X \in K_{0}[t]$ be given by $\bar{\tau}_{i} X=p_{*} \hat{\tau}_{i} \tilde{X}$, where $\tilde{X}$ is any element of $p^{-1}(X)$ (it is well defined since $G$ acts transitively on each fiber) and

$$
\bar{r}_{i} X=\bar{\tau}_{i}(\dot{E}(X)) t-\bar{\tau}_{i}(\Delta X) t^{2} .
$$

Lemma 2.5. Let $p: \tilde{\Gamma} \rightarrow \Gamma$ be a finite covering such that, for all $x, y \in \tilde{\Gamma}_{0}$, all nonzero maps $x \rightarrow y$ in the mesh category of $\tilde{\Gamma}$ have the same depth. Then:

(a) $\tau_{i} X=\bar{\tau}_{i} X(1)$.

(b) $\underline{\underline{P}}_{i}=p^{-1} \underline{\underline{P}}_{i}$.

(c) If $X \in \underline{\underline{P}}_{i}$, then $\bar{r}_{i} X=p_{*} \hat{r}_{i} \tilde{X}$ for any $\tilde{X} \in p^{-1}(X)$ and $r_{i} X=\bar{r}_{i} X(1)$.

Proof. All statements are easily seen to hold for $i=0$. Suppose by induction that the lemma holds for integers less than $i$.

Let $X \in \underline{\underline{P}}_{j}$. If $j \geqslant i$, then $\tilde{X} \in \mathrm{Ob} \underline{\underline{\tilde{C}}}_{i}$ by (b) so $\hat{\tau}_{i} \tilde{X}=\tilde{X}$. Thus $\tau_{i} X=X=p_{*} \tilde{X}=$ $p_{*} \hat{\tau}_{i} \tilde{X}=\bar{\tau}_{i} X=\bar{\tau}_{i} X(1)$. If $j<i$, then

$$
\begin{aligned}
\tau_{i} X & =\tau_{i} r_{j} X \stackrel{(1)}{=} \bar{\tau}_{i}\left(r_{j} X\right)(1) \stackrel{(2)}{=} \bar{\tau}_{i}\left(\bar{r}_{j} X(1)\right)(1) \\
& =\bar{\tau}_{i} \bar{r}_{j} X(1)=p_{*} \hat{\tau}_{i} \hat{r}_{j} \tilde{X}(1)=p_{*} \hat{\tau}_{i} \tilde{X}(1)=\bar{\tau}_{i} X(1) .
\end{aligned}
$$

Here (1) holds by induction on the length of $X$ and (2) holds by induction on $i$. This proves $(\mathrm{a})_{i}$. By 1.6, (a) implies (b) ${ }_{i}$. By (2.4) and 2.2(a), (b) ${ }_{i}$ implies (c) ${ }_{i}$.

THEORLM 2.6. For all artin algebras $\Lambda$ of finite representation type, the paths $I-T t+C t^{2}$ and $h(t)$ from $I$ to $\mathrm{Hom}^{-1}$ are homotopic in $\mathrm{GL}_{n}(\mathbf{R})$ for any $h(t)$ arising from Algorithm 1.13 .

Proof. For each $X \in$ ind $\Lambda$ and $i \geqslant 0$ let $\bar{g}_{i} X=X-\bar{\tau}_{i}(E(X)) t+\bar{\tau}_{i}(\Delta X) t^{2} \in$ $K_{0}[t]$. Let $\bar{M}_{i} \in \mathrm{GL}_{n}(\mathbf{Z}[t])$ be given by $\bar{g}_{i} X=\sum_{Y} \bar{M}_{i}(Y, X) Y$. Then by (2.4) and 2.5 everything works as it did in 2.3. One crucial point is that each component of $\bar{r}_{i} X$ for $X \in \underline{\underline{P}}_{i}$ has length less than the length of $X$. This follows from 2.5(c).

Another consequence of $2.5(\mathrm{~b})$ is the following

Proposition 2.7. Let $p: \tilde{\Gamma} \rightarrow \Gamma$ be any covering map of finite Auslander-Reiten quivers (valued or modulated as defined in [IT2]). Then $p^{-1} \underline{\underline{P}}_{i}=\underline{\underline{\tilde{P}}}_{i}$ for all $i \geqslant 0$.

Proof. Let $p: \Delta \rightarrow \tilde{\Gamma}$ be a covering which satisfies the special condition that for all $x, y \in \Delta$ all nonzero maps $x \rightarrow y$ in mesh $\Delta$ have the same depth. Let $q: \tilde{\tilde{\Gamma}} \rightarrow \Gamma$ be a finite covering such that the composition $\tilde{\tilde{\Gamma}} \stackrel{q}{\rightarrow} \Delta \stackrel{\tilde{p}}{\rightarrow} \tilde{\Gamma} \stackrel{p}{\rightarrow} \Gamma$ is a normal covering. Then $\tilde{\tilde{\Gamma}} \stackrel{\tilde{p} q}{\rightarrow} \tilde{\Gamma}$ is a normal covering and, by $2.5(\mathrm{~b})$,

$$
\underline{\underline{\tilde{P}}}_{i}=\tilde{p} q\left(\underline{\underline{\tilde{P}}}_{i}\right)=\tilde{p} q(p \tilde{p} q)^{-1}\left(\underline{\underline{P}}_{i}\right)=p^{-1}\left(\underline{\underline{P}}_{i}\right) .
$$




\section{REFERENCES}

[A] M. Auslander, unpublished.

[AR] M. Auslander and I. Reiten, Representation theory of artin algebras. VI, Comm. Algebra 6 (1978), 257-300.

[AS] M. Auslander and S. $\emptyset$. Smalø, Preprojective modules over artin algebras, preprint (Trondheim).

$[$ B] $\varnothing$. Bakke, Masters thesis, University of Trondheim.

[DF] P. Donovan and M. R. Freislich, Representable functions on the category of modular representations of a finite group with cyclic Sylow subgroup, J. Algebra 32 (1974), 356-364.

[IT1] K. Igusa and G. Todorov, Radical lavers of representable functors, J. Algebra 89 (1984), 105-147.

[IT2] __ A characterization of finite A uslander-Reiten quivers, J. Algebra 89 (1984), 148-177.

[M] J. Milnor, Introduction to algebraic K-theory, Ann. of Math. Stud., no. 72, Princeton Univ. Press, Princeton, N. J., 1971.

[Ri] C. Riedtmann, Preprojective partitions for selfinjective algebras, J. Algebra 76 (1982), 532-539.

[Ro] B. Rohnes, Preprojective partitions for trivial extensions of hereditary algebras, Comm. Algebra 9 (1983), 949-972.

[T] G. Todorov, A note on preprojective partitions over hereditary artin algebras, Proc. Amer. Math. Soc. 85 (1982), 523-528.

[W1] G. Wilson, The Cartan map on categories of graded modules, J. Algebra 85 (1983), 390-398.

[W2] _ The preprojective partition and Poincare-Betti series for finite dimensional algebras, $\mathrm{Ph} . \mathrm{D}$. Thesis, Brandeis University, 1982.

[WZ] G. Wilson and D. Zacharia, Morphisms between preprojective modules over hereditary artin algebras, Comm. Algebra 11 (1983), 95-107.

[Z1] D. Zacharia, On the Cartan matrix of an artin algebra of global dimension two, J. Algebra 82 (1983), $353-357$.

[Z2] , The preprojective partition of an hereditary algebra, Trans. Amer. Math. Soc. 274 (1982), $327-343$.

Department of Mathematics, Brandeis University, Waltham, Massachusetts 02254

Department of Mathematics, Northeastern University, Boston, Massachusetts 02115 